\title{
ANÁLISE DA ERA CLÍNICA DO TRANSPLANTE INTESTINAL E MULTIVISCERAL
}

\author{
Analysis of the Clinical Era of the Intestinal and Multivisceral Transplantation
}

\author{
Andre Ibrahim David', Fernando Levino ${ }^{2}$, Rogério Carballo Afonso', Ben-Hur Ferraz-Neto'
}

\section{RESUMO}

Introdução: A casuística de doentes tratados com Transplante de Intestino e Multivisceral tem aumentado nos últimos anos. O refinamento de técnicas cirúrgicas, o avanço no tratamento após o transplante e uma melhor compreensão da imunologia do intestino são os principais fatores que levaram este procedimento inicialmente experimental à prática clínica. Além do mais, ocorreram progressos na terapia de imunossupressão, monitoramento e tratamento da rejeição do enxerto, controle das infecções virais e prevenção e tratamento da doença linfoproliferativa pós-transplante. As principais indicações são doenças que levam à Síndrome do Intestino Curto, caracterizadas por má absorção de água e nutrientes em decorrência de extensas ressecções. Em crianças, as principais indicações são: gastroquise (21\%), volvo $(18 \%)$ e enterocolite necrotizante (12\%). Nos adultos, há predominância de complicações vasculares como infarto mesentérico isquêmico ou hemorrágico (22\%), doença de Crohn (13\%) e trauma (12\%). Nessas situações, há necessidade de suporte de vida pela nutrição parenteral. Com o tempo, ocorre trombose dos acessos venosos profundos e infecção do cateter de longa permanência. Objetivo: analisar as características e os resultados dos transplantes de intestino e multivisceral na era clínica. Método: Extensa revisão bibliográfica com artigos indexados no Medline e Lilacs até o mês de janeiro 2009. Resultados: Nos últimos 20 anos, os resultados do Transplante de Intestino e Multivisceral têm apresentado melhora progressiva de sobrevivência em um ano de doentes e enxertos, obtendo-se índices de sucesso de até $80 \%$. A rejeição celular aguda de enxertos intestinais ocorreu mais freqüentemente e com maior gravidade quando comparado a qualquer outro órgão abdominal. Houve um decréscimo significativo na incidência de rejeição grave quando foi usado enxerto multivisceral particularmente em crianças e o uso de terapia de indução de imunossupressão contribuiu para uma diminuição das taxas de rejeição. As principais causas de morte na fase inicial ou tardia de Transplante de Intestino foram sepse e rejeição. Conclusões: No nosso meio, o desenvolvimento do Transplante de Intestino deve ocorrer e ter como objetivo atingir os mesmos resultados internacionais, realidade de outras modalidades de transplante no Brasil.
\end{abstract}

Descritores: Intestino Delgado, Transplante, Rejeição de enxerto, Imunossupressão.

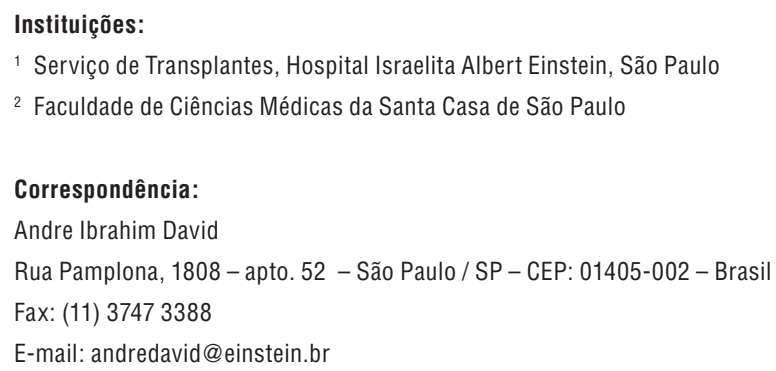

\section{INTRODUÇÃO}

O primeiro transplante de intestino experimental foi realizado por Carrel em 1902. ${ }^{1}$ mas apenas em 1959 Lillehei demonstrou com sucesso um auto-transplante de intestino após preservação fria. ${ }^{2} \mathrm{O}$ transplante multivisceral em caráter experimental foi introduzido por Starzl em 1960. ${ }^{3}$ As primeiras tentativas de realizar transplante de intestino foram mal sucedidas, devido ao que pareciam ser barreiras intransponíveis: rejeição do enxerto e complicações infecciosas. ${ }^{4,5} \mathrm{O}$ primeiro transplante de intestino (TI) realizado com sucesso só ocorreu no final da década de $1980{ }^{6}$

Historicamente, como tratamento clínico, o TI teve evolução mais lenta do que outras formas de transplante de órgãos sólidos. As barreiras começaram a ser entendidas: alta imunogenicidade do intestino, decorrente da elevada carga de tecido linfóide com alto risco de morte por rejeição e a conseqüente infecção pósimunossupressão. As taxas de doença enxerto versus hospedeiro atingiam $15 \%$ e $47 \%$ dos receptores de TI e transplante multivisceral (TMv), respectivamente, em seus primeiros anos de prática clínica (1991-1995). ${ }^{7}$

A casuística dos doentes tratados com Transplante de Intestino e Multivisceral têm aumentado nos últimos anos. O refinamento 
de técnicas cirúrgicas, o avanço no tratamento após o transplante e uma melhor compreensão da imunologia do intestino são os principais fatores que levaram esse procedimento inicialmente experimental à prática clínica. Além do mais, progressos na terapia de imunossupressão, métodos para monitoramento e tratamento da rejeição do enxerto, controle das infecções virais e a prevenção e tratamento da doença linfoproliferativa pós-transplante (DLPT) contribuíram de maneira decisiva para a melhora da sobrevivência no pós-transplante..$^{8,9}$

Apesar disso, esse procedimento continua a ser um dos grandes desafios no campo de transplante de órgãos sólidos, e ainda hoje é um procedimento relativamente incomum, com aproximadamente 1300 transplantes realizados no mundo, sendo $60 \%$ deles em crianças. ${ }^{7}$

\section{INDICAÇÕES E SÍNDROME DO INTESTINO CURTO}

No $8^{\circ}$ Simpósio Internacional de Transplante de Intestino realizado em Miami em 2003, foi definido um consenso que estabelece critérios de referência para o transplante de intestino, ${ }^{10}$ sendo a principal indicação doenças ou intercorrências que levam à síndrome do intestino curto (SIC), caracterizada por má absorção de água e nutrientes em decorrência de extensas ressecções do intestino. ${ }^{8,9,11,12}$ Em crianças, as principais indicações são: gastroquise (21\%), volvo (18\%) e enterocolite necrotizante (12\%). Na população adulta há predominância de indicações vasculares como o infarto mesentérico isquêmico ou hemorrágico $(22 \%)$, doença de Crohn (13\%) e trauma (12\%.) ${ }^{9}$

Muitas das indicações acima estão relacionadas aos grandes ressecados que acabam desenvolvendo a SIC, havendo necessidade de suporte de vida pela nutrição parenteral (NP). Com o tempo, ocorre trombose dos acessos venosos profundos e a principal causa de óbito desses doentes é a sepse, decorrente da infecção do cateter de longa permanência. ${ }^{12}$

Além da alteração anatômica, nos últimos anos foi estabelecido um critério nutricional para definir intestino insuficiente.(12) Recentemente, a citrulina, um aminoácido produzido pelos enterócitos, vem sendo estudada para avaliar a função intestinal. Logo, a medida plasmática de citrulina é uma forma de avaliar pacientes com SIC, atrofia das vilosidades ou rejeição do transplante intestinal. ${ }^{12}$ A citrulina também tem sido estudada na avaliação da evolução do enxerto e sua reabilitação. ${ }^{13,14}$

A NP permite que crianças com SIC tenham crescimento normal durante o longo período de adaptação.(15-18) A NP é associada a drogas e cirurgias para recuperar a função intestinal normal. ${ }^{12}$ No entanto, estudos demonstram a importância da alimentação oral, pois aumenta a secreção gastrintestinal, a secreção do fator de crescimento de epiderme salivar e da motilidade da vesícula biliar. ${ }^{12}$ A alimentação oral torna-se um fator protetor do fígado do paciente e melhora das esteatorréias freqüentes.

Além disso, a NP leva a uma queda na qualidade de vida (QV) dos pacientes. O sucesso atingido com o transplante de intestino delgado como método curativo livrou pacientes de infusões diárias, podendo assim retomar suas atividades diárias sociais e ocupacionais. Em suma, com TI, esses pacientes voltam à alimentação oral, melhor nutrição e redução nas complicações associadas à NP. A melhora da QV ocorre com retorno às atividades familiares, escolares e sociais.

\section{MODALIDADES DE TRANSPLANTE DE INTESTINO}

O transplante de intestino (TI) pode ser realizado de maneira isolada ou em combinação com outros órgãos. O tipo de enxerto é determinado pelas necessidades apresentadas pelo paciente, características da doença, histórico cirúrgico, biótipo do doador e espaço disponível na cavidade abdominal.

No TI, a irrigação vascular é obtida com anastomose arterial na aorta infra-renal, utilizando enxerto vascular de interposição. A drenagem venosa se faz pelo sistema portal, ou, mais freqüentemente, por anastomose na veia cava inferior. ${ }^{19} \mathrm{Na}$ porção terminal do enxerto intestinal realiza-se uma enterostomia de Bishop-Koop, que servirá como estomia diagnóstica; enquanto o cólon do receptor é anastomosado ao enxerto $20 \mathrm{~cm}$ proximalmente a essa estomia, fazendo-se uma íleo-colostomia latero-lateral. Quando se trata de doadores vivos ou em casos em que há grande desproporção entre o tamanho e o peso do doador falecido e do receptor, transplanta-se um segmento de $200 \mathrm{~cm}^{.20}$

Devem ser preservadas porções viáveis do intestino original, já que dados recentes sugerem que o intestino remanescente é fator de proteção contra a nutrição por via parenteral, que poderá ser necessária por um certo período de tempo após o TI, além do intestino residual aumentar a chance de sucesso de uma nova jejunostomia ou de uma anastomose diretamente ao cólon remanescente caso haja falência do enxerto.

O transplante de fígado e intestino (TFI), modalidade mais comumente utilizada para tratamento de crianças, pode ser realizado em bloco ou separadamente. ${ }^{21}$ Quando em separado, os dois órgãos podem ser transplantados simultaneamente ou seqüencialmente, sendo do mesmo doador ou de doadores diferentes, lembrando que há possibilidade de realização de TFI com doador vivo, principalmente para receptores pediátricos. Quando em bloco, transplanta-se também o duodeno e um segmento ou a totalidade do pâncreas, para evitar torção do eixo portal e necessidade de reconstrução biliar. A drenagem é feita por anastomose porto-cava término-lateral. ${ }^{22}$

O transplante multivisceral é definido como a remoção e transplantação de todas as porções do intestino delgado e estômago, associados ou não a outros órgãos abdominais. ${ }^{23}$ Enxertos que não contêm estômago não são classificados como multiviscerais, de acordo com o Registro de Transplante de Intestino (ITR); a presença do fígado, no entanto, é opcional. Nesse tipo de transplante, o estômago, o complexo pancreato-duodenal, o intestino delgado e outras vísceras abdominais, dependendo das necessidades clínicas do receptor, são ressecados e transplantados em bloco. Um segmento da aorta do doador que contém o tronco celíaco e a artéria mesentérica superior é anastomosado na aorta do receptor a Carrel ou de maneira término-lateral, criando um neotronco celíaco. A drenagem venosa depende se haverá ou não transplante hepático concomitante. Se o fígado for incluído no TMv, a drenagem venosa de todo enxerto é feita por Piggy-back ou por interposição da veia cava retro-hepática. Se não houver inclusão do fígado no enxerto, a drenagem venosa se dará pelo sistema porta ou pela veia cava inferior. Os rins, glândulas supra-renais e cólon do doador podem ser incluídos no enxerto, dependendo de sua necessidade clínica. ${ }^{24}$

Uma técnica modificada de TMv com preservação de órgãos foi recentemente proposta por dois grupos. ${ }^{25,26}$ Nessa técnica, o pâncreas e o baço nativo são preservados, sua drenagem venosa é feita por um shunt portocaval e a drenagem exócrina pancreática 
é redirecionada ao jejuno do enxerto. Com seu uso, observou-se que o risco de falência pancreática, doença linfoproliferativa póstransplante e sepse pós-esplenectomia diminuía consideravelmente. Há, no entanto, uma dúvida em relação a sua nomenclatura. Como a nomenclatura dos transplantes contendo intestino é baseada no tipo e número de órgãos no enxerto e não nos órgãos ressecados, argumenta-se que a preservação espleno-pancreática não deveria ser classificada como TMv modificado. Em receptores adultos de TMv houve melhor sobrevivência do enxerto em 14 pacientes submetidos a TMv modificado com preservação espleno-pancreática, em comparação com um grupo de 11 pacientes submetidos à técnica tradicional de TMv com espleno-pancreatectomia. A preservação do baço e do pâncreas originais favoreceu o receptor com menos complicações infecciosas graves, casos de DLPT, doença do enxerto versus hopedeiro $(\mathrm{DEvH})$, insuficiência pancreática endócrina e exócrina pós-transplante. No grupo em que os receptores foram submetidos a espleno-pancreatectomia houve dois casos de DLPT e dois de DEvH, além de grande número de infecções pósesplenectomia, que foram a maior causa de óbito. ${ }^{26}$

Um dos problemas mais desafiadores em pacientes com SIC são os danos à parede abdominal e à cavidade abdominal decorrentes de múltiplos procedimentos cirúrgicos prévios. Sendo assim, o fechamento primário da cavidade abdominal após o transplante nem sempre é possível, devido a "perda de domicílio". ${ }^{27}$ Uma análise recente identificou que a correlação entre o peso do receptor e do doador é o fator mais importante para determinar problemas de fechamento da parede abdominal. ${ }^{28}$ A dificuldade do fechamento da parede abdominal pode ter graves conseqüências. A cavidade muito apertada pode levar à Síndrome Compartimental Abdominal, à parede aberta coberta por "mash", ao aumento do risco de infecções intracavitárias e a lesões do enxerto, assim como complicações respiratórias. A taxa de re-transplante é maior nesse grupo. ${ }^{27}$

Não há solução ideal para evitar ou tratar defeitos da parede abdominal decorrentes de TI. Esses defeitos podem ser tratados com o uso de enxertos prostéticos, próteses de Silastic, enxertos de fáscia muscular do mesmo doador falecido ou matriz acelular dermóide. ${ }^{29}$ Recentemente, o transplante de parede abdominal foi proposto como alternativa terapêutica. 27

\section{RESULTADOS}

Nos últimos 20 anos tem havido melhora progressiva nos resultados da sobrevivência em um ano de pacientes e enxertos, obtendo-se índices de sucesso de até $80 \%$. $^{70} \mathrm{O}$ aumento do uso de terapias de indução associado à estratégia de transplante precoce, feito antes do aparecimento de complicações relacionadas a nutrição parenteral, constituem os principais fatores para o sucesso do TI. Houve aumento da sobrevivência em curto prazo, porém, a sobrevivência de longo prazo ainda não apresentou melhora significativa. Os argumentos são que a quantidade de imunossupressão e a rejeição crônica do enxerto, assim como o início tardio da rejeição aguda, ainda representam desafios para o incremento dos resultados em longo prazo do TI. Uma percepção mais aprofundada da análise dos registros é que a presença do fígado no enxerto aumenta a sobrevivência. ${ }^{8,9}$

Com a introdução do tacrolimo como droga imunossupressora a partir 1990, o número de TI cresceu progressivamente. A terapia imunossupressora de indução em diferentes períodos de tempo devido ao alto risco de rejeição precoce e tardia do enxerto é usada em $90 \%$ dos casos, sendo mais comumente realizada com o uso de anticorpos monoclonais anti-receptor de interleucina 2. Avanços significativos na sobrevivência do enxerto em longo prazo e a redução da toxicidade associada ao tacrolimo podem ser obtidos com o uso de sirolimo.

As causas de morte na fase inicial ou tardia do TI são principalmente sepse e rejeição ${ }^{7}$. A doença linfoproliferativa pós-transplante (DLPT), no entanto, predomina na fase tardia do TI. Sua freqüente manifestação no enxerto intestinal constitui um grande problema, já que o diagnóstico diferencial com rejeição muitas vezes é bastante dificultado. O prognóstico da DLPT tem melhorado drasticamente com o uso de rituximab. ${ }^{31}$

A rejeição celular aguda (RCA) de enxertos intestinais ocorre mais freqüentemente e com maior gravidade quando comparado a qualquer outro órgão abdominal. ${ }^{32}$ A provável razão é a elevada imunogenicidade do enxerto. Embora a RCA seja atualmente identificável pela biópsia intestinal, ${ }^{32,} 33$ ela é menos entendida do que outros órgãos sólidos. Existe um decréscimo significativo na incidência de rejeição grave quando é usado enxerto multivisceral, particularmente em crianças. Autores ainda mostram que o uso rotineiro de terapia de indução de imunossupressão contribuiu para uma diminuição das taxas de rejeição, , 9, 34

Dentre doentes que apresentaram rejeição, o primeiro episódio ocorreu no intervalo médio de duas semanas e meia após o transplante, reforçando a necessidade de realizar frequentes endoscopias e avaliações histológicas do enxerto nos primeiros meses após o TI. Como o número total de episódios de rejeição diminui com o passar do tempo e na maior parte ocorre antes do primeiro ano após o transplante, a freqüência da monitoração pode então ser gradativamente reduzida. A ocorrência de rejeição leve a moderada não tem efeito significativo na sobrevivência do enxerto; entretanto, duas variáveis críticas que afetam a sobrevivência do enxerto foram identificadas: progressão para rejeição grave e duração da rejeição acima de 21 dias. Selvaggi e col., concluíram que ambos devem ser evitados iniciando terapia esteróide em bolus precoce e agressivamente, assim como expandindo a terapia a agentes anti-linfócitos em caso de ausência de resposta aos esteróides em no máximo 2-3 dias. ${ }^{35}$

Os avanços das estratégias de monitoração podem em alguns casos tornar desnecessária a realização de biópsias do enxerto (36) ou ajudar a identificar possíveis locais de rejeição. Marcadores como a citrulina e a calprotectina ${ }^{37,38}$ são promessas como medidas de alteração da função do enxerto. Em uma análise multivariada de RCA leve, moderada ou grave, bacteremia ou infecção respiratória, idade pediátrica e tempo entre o TI e a coleta da amostra de sangue foram identificados como fatores associados a níveis mais baixos de citrulina. No entanto, a citrulina plasmática, quando são afastadas outras etilogias, reflete somente a extensão da lesão mucosa, independentemente de sua etiologia. ${ }^{39}$

As infecções virais são uma área importante e de preocupação em TI. Avanços na profilaxia e vigilância às infecções virais e a prevenção e tratamento de DLPT por vírus de Epstein-Barr (EBV) contribuíram para a melhora do resultado do enxerto. A profilaxia viral pode ser realizada com ganciclovir, valganciclovir, e hiperimunoglobulina de citomegalovírus (CMV). Rotineiramente, a vigilância antiviral consiste em monitorar a evidência viral, particularmente DNA de CMV e EBV e antígeno pp65 de CMV em sangue ou tecidos.(40) Níveis altos de DNA de EBV podem, por exemplo, ser um prelúdio de DLPT. $(41,42) \mathrm{O}$ monitoramento de rotina para adenovírus e 
outros enterovírus é feita com menor frequência. Além da terapia antiviral, a redução precoce da imunossupressão em torno de 20$50 \%$ é também prática comum. ${ }^{43}$

A doença enxerto versus hospedeiro (DEvH) tem grande importância em TI isolado ou combinado desde o início da realização do procedimento. De acordo com o Intestinal Transplant Registry (ITR), a incidência de DEvH era de $47 \%$ em TMv e de aproximadamente $15 \%$ em TFI até 1995 . Após essa data, a incidência diminuiu para $8 \%$ em TI e aproximadamente $12 \%$ em TMv e TFI. Após 2000, a incidência média era de 7-8\%. ${ }^{7,44,45}$

\section{QUALIDADE DE VIDA E CUSTO-BENEFÍCIO}

Em trabalho recente de revisão, Sudan e col. apontam que devido ao pequeno número de estudos e à natureza preliminar dos achados, não podem ser tiradas conclusões a respeito da QV após TI. No entanto, os dados disponíveis são encorajadores, sugerindo que a QV foi relativamente boa após o TI, e talvez semelhante à de indivíduos normais.

Uma visão geral pode ser obtida pelos dados de QV do International Intestinal Transplant Registry, em que são registradas impressões de médicos e enfermeiras. ${ }^{7}$ 85\% dos receptores de TI obtiveram escore de Karnofsky de 90-100\% mais do que seis meses após o transplante. Esses dados sugerem que a maioria dos receptores de TI tem uma ótima QV. No entanto, deve-se considerar que as impressões não são dos receptores, mas de seus cuidadores.

Outros estudos mostram que a maioria dos doentes estava completamente fora da NP após o TI, e tiveram baixo número de hospitalizações e complicações no primeiro ano pós-TI. Os doentes têm boa reabilitação social e profissional, sendo que 50\% voltaram a trabalhar após o TI. ${ }^{46,47}$

Temos que dar valor necessário ao aspecto econômico. O custo médio do TI isolado foi estimado independentemente pelos grupos de Pittsburgh (1994-1998) e de Omaha (2002-2003), sendo estimado aproximadamente de US\$132.000 a 135.000.(48) Levando em consideração os custos de re-hospitalização de US\$ 9.000 - 23.500 por ano após TI e em comparação aos custos de US\$ 100.000 a 150.000 da NP, o TI tem maior custo-benefício dois anos após o procedimento.

\section{CONCLUSÃO}

O TI é a única terapia definitiva para falência intestinal irreversível, com melhora progressiva da sobrevivência do doente e do enxerto em curto e longo prazo, com indicação na falência da NP devido a perda de acessos vasculares e infecções. As razões para a melhora do TI são multifatoriais. No entanto, a melhora da imunossupressão certamente tem o papel mais importante, particularmente a implementação de drogas de indução, que aumentaram as taxas de sobrevivência do enxerto em um ano para cerca de $80-90 \%$. Os avanços no TI também se refletem por uma considerável redução nas taxas de rejeição. As rejeições moderadas diminuíram de 65\% para pouco mais de $20 \%$, e as rejeições graves têm se mantido em torno de $10 \%$ nos últimos oito anos, mas ainda representando um dos principais riscos de falência do enxerto.

Dados sobre a influência do estado do receptor antes de se submeter ao TI mostram claramente a necessidade da avaliação precoce dos doentes com falência intestinal nos centros de referência, antes que haja complicações que ameacem a vida do doente. Com uma maior informação sobre os critérios de indicação do TI e do tempo de realização da cirurgia, serão obtidos melhores resultados.

Como as taxas atuais de sobrevivência do TI são equivalentes à terapia bem sucedida de NP prolongada, o transplante deverá se tornar em breve a terapia de escolha, e não somente terapia complementar para indivíduos com falência intestinal. Em nosso meio, o desenvolvimento do TI deve ocorrer e ter como objetivo atingir os mesmos resultados internacionais, realidade de outras modalidades de transplante no Brasil. ${ }^{49}$

\section{ABSTRACT}

Introduction: Nowadays, there has been an increase in the casuistic of intestinal and multivisceral transplant. Surgical technical improvements, close post transplant follow-up and better understanding of the intestinal immunology are the main reasons for this classic experimental research to the clinical practice. Furthermore, there is an improvement in the immunosuppressant therapy, in the graft rejection diagnosis, in the post-transplant linfoproliferative disease therapy and in viral infection monitoring and treatment. The post-extensive intestinal resection short gut Syndrome with later very poor absorption of water and nutrients is the classic indication to intestinal transplant. Children's major indications are: gastroschise (21\%), volvulus (18\%) and necrotizing enterocolitis (12\%). The adult's are: vascular diseases such as ischemic or hemorrhagic mesenteric stroke (22\%), Crohn disease (13\%) and trauma (12\%). Parenteral nutrition is the life support for suxh sicknesses, but with serious complications such as deep vascular access thrombosis and catheter infection. Purpose: to analyze the characteristics and results for the intestinal and multivisceral transplant in the clinical era. Methods: Extensive literature review of articles indexed in Medline and Lilacs until January, 2009. Results: In the past 20 years, the results of the intestine and multivisceral transplantation have shown a progressive survival improvement at one year both for patients and grafts, attaining success rates of up to $80 \%$. Acute cellular rejection of intestinal grafts occurred more frequently and with greater severity when compared to any other abdominal organ. There was a significant decrease in the incidence of severe rejection upon the use of multivisceral graft, particularly in children, and the use of the therapy to induce the immunosuppression has contributed to a decrease in the rejection rates. The major causes of death in the early or late intestine transplantation were sepsis and rejection. Conclusions: In Brazil, the development of the intestine transplantation should occur, having as a goal to achieve the same results attained in international centers, a reality already attained by other modalities of transplantation in Brazil.

Keywords: Small bowel, Transplantation, Graft Rejection, Imunossupression 


\section{REFERÊNCIAS}

1. Carrel A. La technique operatoire des anastomoses vasculaires et la transplantation des visceres. . Lyon Med; 1902. p. 859-63.

2. Lillehei RC, Goott B, Miller FA. The physiological response of the small bowel of the dog to ischemia including prolonged in vitro preservation of the small bowel with successful replacement and survival. Ann Surg. 1959;150:543-60.

3. Starzl TE, Kaupp HA Jr. Mass homotransplantation of abdominal organs in dogs. Surg Forum. 1960;11:28-30.

4. Lillehei RC, Idezuki Y, Feemster JA, et al. Transplantation of stomach, intestine, and pancreas: experimental and clinical observations. Surgery. 1967;62(4):721-41.

5. Okumura M, Mester M. The coming of age of small bowel transplantation: a historical perspective. Transplant Proc. 1992;24(3):1241-2.

6. Grant D, Abu-Elmagd K, Reyes J, et al. 2003 report of the Intestine Transplant Registry: A new era has dawned. Annals of Surgery. 2005;241(4):607-13.

7. Intestinal Transplant Registry data. 2003

8. Kato T, Tzakis AG, Gennaro S, et al. Intestinal and multivisceral transplantation in children. Annals of Surgery. 2006;243(6):756-66.

9. Tzakis AG Kato T LD, et al. 100 multivisceral transplants at a single center. Annals of Surgery. 2005;242(4):480-90.

10. Ruiz P BA, Reyes J, et al. Histological criteria for the identification of acute cellular rejection in human small bowel allografts: Results of the Pathology Workshop at the VIII International Small Bowel Transplant Symposium. Transplantation Proceedings. 2004;36:335-7.

11. Abu-Elmagd K, Reyes J, Bond G, et al. Clinical intestinal transplantation: a decade of experience at a single center. Ann Surg. 2001;234(3):404-17.

12. Goulet O, Sauvat F. Short bowel syndrome and intestinal transplantation in children. Curr Opin Clin Nutr Metab Care. 2006 9(3):304-13.

13. David AI Gaynor JJ ZP, et al. . An association of lower serum citrulline levels within 30 days of acute rejection in patients following small intestine transplantation. Transplantation Proceedings. 2006.

14. David AI SG, Ruiz P, et al. . Blood citrulline level is an exclusionary marker for significant acute rejection after intestinal transplantation. Transplantation. 2007;84:1077.

15. Goulet O, Révillon Y, Jan D, et al. Neonatal short bowel syndrome. J Pediatr. 1991;119(1):18-23.

16. Sondheimer JM CM, Sontag M, Zerbe GO. Predicting the duration of dependence on PN after neonatal intestinal resection. J Pediatr. 1998;132:80-4.

17. Leonberg BL CE, Eicher P, et al. Long-term growth and development in children after home PN. J Pediatr. 1998;132:461-6.

18. Festen S BJ, Goldhoorn GA, et al. ; :. Excellent long-term outcome for survivors of apple peel atresia. J Pediatr Surg. 2002;37:61-5.

19. Berney T, Kato T, Nishida S, et al. Portal versus systemic drainage of small bowel allografts: comparative assessment of survival, function, rejection, and bacterial translocation. J Am Coll Surg. 2002;195(6):804-13.

20. Kato T, Gaynor JJ, Selvaggi G, et al. Intestinal transplantation in children: A summary of clinical outcomes and prognostic factors in 108 patients from a single center. Journal of Gastrointestinal Surgery. 2005;9(1):75-89. discussion

21. Fishbein T, Florman S, Gondolesi G, Decker R. Noncomposite simultaneous liver and intestinal transplantation. Transplantation. 2003;75(4):564-5.

22. Gondolesi GE, Rodriguez-Davalos M, Soltys K, Florman S, Kaufman S, Fishbein T. End-to-end portocaval shunt for venous drainage of the native foregut in combined liver-intestinal transplantation. Pediatr Transplant. 2006;10(1):98-100.

23. Starzl TE, Todo S, Tzakis A, et al. The many faces of multivisceral transplantation. Surg Gynecol Obstet. 1991;172(5):335-44.
24. Pascher A, Klupp J, Kohler S, Langrehr JM, Neuhaus P, et al. Transplantation of an eight-organ multivisceral graft in a patient with frozen abdomen after complicated Crohn's disease. World J Gastroenterol. 2006;12(27):4431-4.

25. Matsumoto CS FT. Modified multivisceral transplantation with splenopancreatic preservation. Transplantation. 2007;83(2):234-6.

26. Abu-Elmagd KM. Preservation of the native spleen, duodenum, and pancreas in patients with multivisceral transplantation: nomenclature, dispute of origin, and proof of premise. Transplantation. 2007;84(9):1208.

27. Levi DM, Tzakis AG, Kato T, et al. Transplantation of the abdominal wall. Lancet. 2003;361(9376):2173-6.

28. Carlsen BT, Farmer DG, Busuttil RW, Miller TA, Rudkin GH. Incidence and management of abdominal wall defects after intestinal and multivisceral transplantation. Plast Reconstr Surg. 2007;119(4):1247-55.

29. Asham E, Uknis ME, Rastellini C, Elias G, Cicalese L. Acellular dermal matrix provides a good options for abdominal wall closure following small bowel transplantation: a case report. Transplant Proc. 2006;38(6):1770-1.

30. Grant D A-EK, Reyes J, et al., . On behalf of the Intestine Transplant Registry. 2003 report of the intestine transplant registry: a new era has dawned. Ann Surg. 2005.

31. Nishida S, Kato T, Burney T, et al. Rituximab treatment for posttransplantation lymphoproliferative disorder after small bowel transplantation. Transplant Proc. 2002;34(3):957.

32. Garcia M, Delacruz V, Ortiz R, et al. Acute cellular rejection grading scheme for human gastric allografts. Hum Pathol.2004;35(3):343-9.

33. Wu T, Abu-Elmagd K, Bond G, Nalesnik MA, Randhawa P, Demetris AJ. A schema for histologic grading of small intestine allograft acute rejection. Transplantation. 2003;75(8):1241-8.

34. Tzakis AG, Kato T, Nishida S, et al. Alemtuzumab (Campath- 1H) combined with tacrolimus in intestinal and multivisceral transplantation. Transplantation. 2003;75(9):1512-7.

35. Selvaggi G, Gaynor JJ, Moon J, et al. Analysis of acute cellular rejection episodes in recipients of primary intestinal transplantation: a single center, 11-year experience. Am J Transplant. 2007;7(5):1249-57.

36. Ruiz P, Garcia M, Pappas $P$, et al. Mucosal vascular alterations in isolated small-bowel allografts: relationship to humoral sensitization. Am J Transplant. 2003;3(1):43-9.

37. Pappas PA, Tzakis A, Gaynor JJ, Carreno MR, Ruiz P, Frans Huijing, et al. An analysis of the association between serum citrulline and acute rejection among 26 recipients of intestinal transplant. Am J Transplant. 2004;4(7):1124-32.

38. Fagerberg UL, Loof L, Myrdal U, Hansson LO, Finkel Y. Colorectal inflammation is well predicted by fecal calprotectin in children with gastrointestinal symptoms. J Pediatr Gastroenterol Nutr. 2005;40(4):450-5.

39. David AI, Selvaggi G, Ruiz P, et al. Blood citrulline level is an exclusionary marker for significant acute rejection after intestinal transplantation. Transplantation. 2007;84(9):1077-81.

40. Horslen SP. Optimal management of the post-intestinal transplant patient. Gastroenterology. 2006;130(2 Suppl 1):S163-9.

41. Rowe DT, Qu L, Reyes J, et al. Use of quantitative competitive PCR to measure Epstein-Barr virus genome load in the peripheral blood of pediatric transplant patients with lymphoproliferative disorders. J Clin Microbiol. 1997;35(6):1612-5.

42. Kogan-Liberman D, Burroughs M, Emre S, Moscona S, Shneider BL. The role of quantitative Epstein-Barr vírus polymerase chain reaction and preemptive immunosuppression reduction in pediatric liver transplantation a preliminary experience. J Pediatr Gastroenterol Nutr. 2001;33(4):445-9.

43. Serinet MO, Jacquemin E, Habes D, Debray D, Fabre M, Bernard O. Anti-CD20 monoclonal antibody (Rituximab) treatment for Epstein-Barr virus-associated, B-cell lymphoproliferative disease in pediatric liver transplant recipients. J Pediatr Gastroenterol Nutr. 2002;34(4):389-93. 